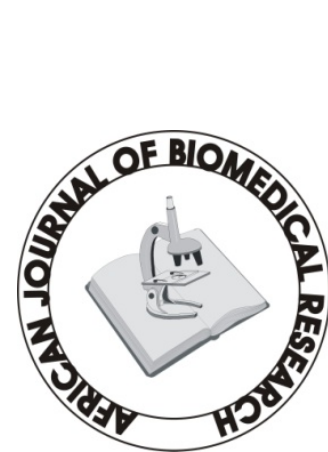

Full-text available at http://www.ajbrui.com http://www.bioline.br/md http://www.ajol.com

Received:

October 2007

Accepted (Revised): December 2007

Published

May 2008
Mini -Review

\section{Pain in Osteoarthritis}

\section{${ }^{1}$ Enohumah KO and ${ }^{2}$ Imarengiaye $\mathrm{CO}$}

${ }^{1}$ Department of Anaesthesiology, Our Lady of Lourdes Hospital

Drogheda, IRELAND

${ }^{2}$ Department of Anaesthesiology, University of Benin Teaching Hospital, Benin City, Edo State. NIGERIA

\section{ABSTRACT}

Osteoarthritis (OA) has been described "as a condition characterized by userelated joint pain experienced on most days in any given month, for which no other cause is apparent”. The primary problem in OA is the damage to the articular cartilage which triggers a series of other events that culminate in pain and loss/limitation of function in the affected joint. OA is estimated to affect $70 \%$ to $80 \%$ of people older than 55 years. There is a vast body of evidence to suggest that $\mathrm{OA}$ is a heterogenous condition that involved not only the articular cartilage but also an adaptive response of the bone and the synovium to a variety of environmental, genetic and biomechanical stresses. Undoubtedly, pain which is the most prominent and disabling presentation of $\mathrm{OA}$ is an increasingly important public health problem especially with an increasing aging population.

(Afr. J. Biomed. Res. 11: 119 - 128)

Key Words; arthritis, pain, economic impart, nociception, hyperalgesia

*Address for Correspondence : enohumah@yahoo.com; Phone-+353-874126805 


\section{INTRODUCTION}

Osteoarthritis (OA) has been described "as a condition characterized by use-related joint pain experienced on most days in any given month, for which no other cause is apparent” (Agel et al 2000). The pathological changes seen in OA is characterized by focal areas of loss of articular cartilage within the synovial joints, associated with hypertrophy of the bone (osteophtes and subchondral sclerosis) and thickening of the capsule (Agel et al 2000). Rehman and Lane in 1999 described OA as a chronic, degenerative disease associated with joint pain and loss of function. The primary problem in $\mathrm{OA}$ is the damage to the articular cartilage which triggers a series of other events that culminate in pain and loss/limitation of function in the affected joint. In The global burden of disease and The burden of musculoskeletal conditions at the start of the new millennium published by World Health Organisation (WHO), OA is ranked fourth in health impact in women and eighth in men in the western world (Murray 1996, Agel et al 2000). OA is estimated to affect $70 \%$ to $80 \%$ of people older than 55 years. In England and Wales, between 1.3 and 1.75 million people have symptomatic OA (Reginster 2002). Over half a million people in the UK have severe knee OA while 80,000 hip or knee replacements were performed in 2000 in the UK with a cost of $£ 405$ million. Undoubtedly, pain which is the most prominent and disabling presentation of $\mathrm{OA}$ is an increasingly important public health problem especially with an increasing aging population (Murray1996).

The aims of this review are to evaluate in physiological terms the mechanisms involved in the initiation of pain in OA, the peripheral and central mechanisms involved in pain transduction, transmission, perception and modulation. Secondly, the mechanism involved in the progression of OA pain becoming chronic pain
(CP) will be reviewed.

A literature search was conducted using the keywords arthritis, pain, economic impart, nociception, hyperalgesia and long-term potentiation.

Several research papers were published in the late 1990's and 2000's detailing the results of studies conducted to establish the possible mechanisms of the pathophysiology of $\mathrm{OA}$ and the methods involved in the destruction of the involved joints (Denko et al 1996, Pelletier et al 1996, Westacott \& Sharif 1996, Fink et al 2001, Volck et al 2001, Yuan et al, 2004, Shibakawa et al 2005). In addition several animal research papers were also published in the last decade aimed at understanding the pain processes in OA. Few human research papers are however available describing the possible physiological mechanism involved in the process of pain mechanism in OA. This review was therefore based on computerized search of relevant papers from 2000-2005 in the EMBASE/ Medline. Due to lack of sufficient research papers on the mechanism of pain in OA and progression to $\mathrm{CP}$ relevant papers outside these dates were included in the review to ensure the most up-to-date research available.

To ensure clarity and in keeping with the aims, this literature review will be divided into six sections.

These include

- Anatomy of the joint

- Sources of nociception in the joint

- Pathophysiology of OA

- Mechanism of pain in OA

- Progression of OA to CP

- Implications for practice

\section{Anatomy of a joint}

A brief review of the basic anatomy of a typical synovial joint is presented here to help understand the mechanisms involved in OA-induced damages of the involved joint which culminate in pain and other symptoms of OA.

A joint is where two bones meet. Articular cartilage covers the bone ends which are lubricated by synovial fluid. The cartilage is made up of $70-80 \%$ of water and a type II collagen with 
proteoglycans and glycosaminoglycans produced by chrondrocytes. The collagen fibres in the cartilage offer tensile strength to the cartilage because of its architectural makeup. The cartilage however contains no intrinsic blood vessels. It receives its nutrition from the synovial fluid. The synovial fluid, which is secreted by the synovial membrane lining the inner surface of the joint, facilitates not only movement but also provides nutrients, phagocytosis and other immunologic functions within the joint (Romanes 1986, Martini 1998, Snell 2004). The integrity of a joint is therefore dependent upon its architecture, the cartilage, bone and the supporting structures enclosing the joint. OA in simple terms is a result of alterations in the aforementioned architectural structures within the joint with a resultant pain, loss of function and instability in the involved joint. Figure 1 shows the diagram of a typical synovial joint.

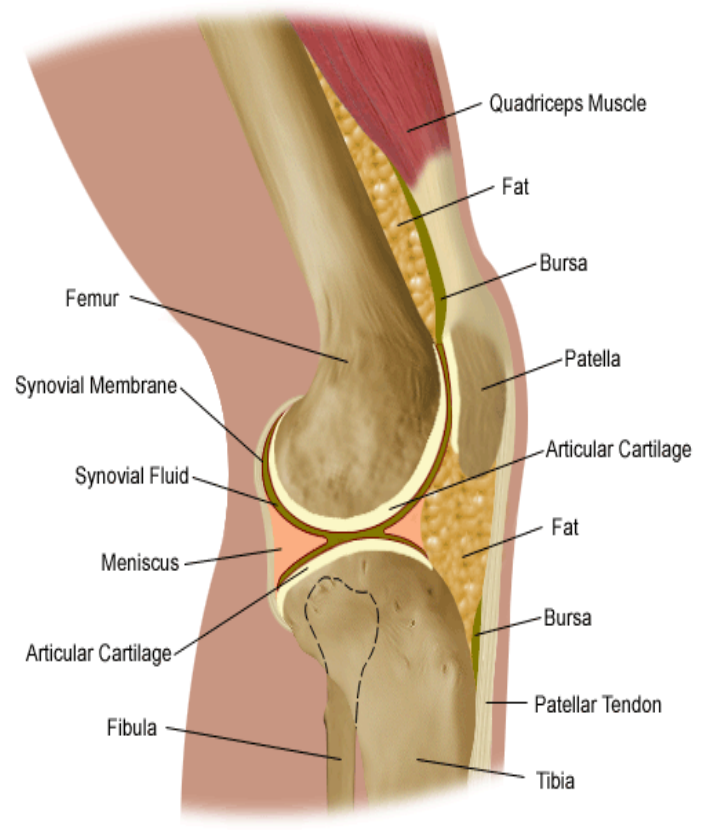

Fig. 1: Anatomy of the knee

\section{Sources of nociception in the joint}

The term "nociception" was coined by the Nobel Laureate Sherrington (1960) to designate a physiological sensory phenomenon. Also "nociception" is derived from "nocere" the Latin word for to hurt. Nociceptors on the other hand are peripheral sensory organs which are activated when a nociceptive stimuli cause tissue damage. These nociceptors are unspecialized, naked nerve endings found close to small blood vessels and mast cells. The functional nociceptive unit is therefore made up of the structural triad of capillary, nociceptor and mast cell. This is the unit that is sensitive to tissue damage (Berne \& Levy 1998, Ganong 2001).

Recent evidence (Wyke 1981, Alvarez \& Fyffe 2000, Lawson 2002) indicate that four different types of nerves innervate the joint and there is a rich supply of myelinated and unmyelinated fibers innervating the joint capsule, subchondral bone, periosteum, ligaments and menisci. These four fibers include Type $1(\mathrm{~A} \alpha)$, Type $2(\mathrm{~A} \beta)$, Type 3 (A $\delta)$ and Type 4 (C). Wyke (1981) in a review of neurology of joint from animal studies indicates that types 3 and 4 have been found in most joint structures with the exception of articular cartilage. Another study by Wojtys and colleagues in 1990 demonstrated that the periosteum, pad fat deep to patella, subchondral bone and joint capsule are made up of fibres containing substance P (SP). SP was not demonstrated in the articular cartilage in this study. Two further studies (Kellgren \& Samuel 1950, Dye \& Chew 1993) using awake patients scheduled for arthrotomy or arthroscopy of the knee shown that most pain-sensitive structures are located in the pad fat deep to patella, ligaments and synovium. The cartilage was not tender in the subjects recruited for this study suggesting that pain-sensitive fibres are not present in the articular cartilage.

In the anatomy of the joint described above, the cartilage does not contain blood vessels but derives it nutrients from the synovium. Thus, whatever its role in the pathogenesis of joint damage, it cannot be the tissue OA pain originate. The subchondral bone, periosteum, synovium, ligaments, and the joint capsule contain nerve endings that could be the source of nociceptive stimuli in OA (Creamer et al 1996, Felson et al 2003). Irritation of the periostal as a result of remodeling, denuded bone, compression of soft 
tissue by osteophytes, microfractures of the subchondral bone, effusion and spasm of surrounding muscles has been shown to contribute to the pain that may be felt by patients with OA (Townes 1999).

Felson et al (2001) in a cross-sectional observational study of 401 patients with demonstrable radiological knee OA found a significant $(p<0.001)$ bone marrow lesion in persons with painful knee (77.5\%) compared with $30 \%$ of persons without pain. Again large lesions were present almost exclusively in patients with knee pain compared with persons without pain $(p<0.001)$. They concluded that bone marrow lesions on MRI are strongly associated with the presence of pain in knee OA. Also, Sowers et al (2003) in a study of women in their 40s with and without knee pain showed that, whereas tiny bone marrow lesions were not associated with pain, larger lesions similar to those identified by Felson et al (2001) were strongly correlated with the presence of knee pain.

These two findings suggest that the bone in the periosteum and bone marrow is richly innervated with nociceptive fibres and represent a potential source of nociceptive pain in patients with OA.

\section{Pathophysiology of OA}

Significant advances have been made in understanding the pathophysiology of OA. A large body of clinical evidence has demonstrated the gradual proteolytic degradation of the joint cartilage matrix in OA. Metalloproteinases produced catalyze this process. The synovium has also been shown to be inflamed in OA. Beside the inflammation of the synovium, the synovium has equally be linked with the production of high levels of interleukin-1 (IL-I), tumour necrosis factor- $\alpha$ (TNF- $\alpha$ ), and cytokines. Cytokines and the other substances released from the cartilage, synovium and the bone invariably affect the chondrocyte function and when catabolism exceeds cartilage synthesis, osteoarthritis develops (Denko et al 1996, Pelletier et al 1996, Westacott \& Sharif 1996, Fink et al 2001, Volck et al 2001, Yuan et al, 2004, Shibakawa et al 2005). These complex series of events result in the early changes seen in OA as outlined below:

\section{Stages of OA (Martel-Pelletier, 2004)}

Stage I: There is proteolytic breakdown of cartilage matrix

Stage II : There is fibrillation and erosion of cartilage surface, accompanied by the release of breakdown products into the synovial fluid

Stage III: Synovial inflammation begins when synovial cells ingest a breakdown product through phagocytosis and produce proteases and proinflammatory cytokines.

\section{Radiological changes in OA (Haq et al, 2003)}

- Joint space narrowing

- Osteophytes

- Bony cysts

- Subchondral sclerosis

Inflammation pathway which hitherto was not considered as contributory to the pathogenesis of OA has been shown to be involved in OA. Advances in detection methods have made it possible to demonstrate that the inflammatory pathways are upregulated in OA (Brooks 2003, van den Berg 2003, Abramson 2004).

Recently, the role of Nitric oxide (NO) in the pathogenesis of $\mathrm{OA}$ and in mechanical signal transduction has been described (Pelletier J-P et al 1996, Rawlinson et al 1996, Fink et al 2001). Cartilage from patients with rheumatoid arthritis and osteoarthritis spontaneously produces NO in vitro. In experimental osteoarthritis, NO induces chondrocyte apoptosis, thus contributing to cartilage damage. These findings suggest that unregulated NO production in humans play a part in the pathophysiology of OA.

In addition to these structural damages in the involved joint, psychological factors, muscle weakness and comorbidities may play roles in the degree of the eventual pain felt by patient with OA.

Risk factors for OA. (Kraus, 1997)

1. Age older than 50

2. Crystals in joint fluid or cartilage

3. High bone mineral density

4. History of immobilistaion

5. Injury to the joint

6. Joint hypermobility or instability

7. Obesity (weight-bearing joints) 
8. Periperal neuropathy

9. Prolonged occupational or sports stress

The classification of $\mathrm{OA}$ as primary and secondary are shown below

\section{Classification of OA (Birchfield, 2001)}

Primary OA Has no known cause. Common. Related to aging and hereditary. May be localized or generalized. Commonly affects the distal interphalangeal joints of the hands, hip and the knee. The cervical and lumbar spine may be affected.

Secondary OA Causes include articular injury, obesity, paget's disease, or inflammatory arthritis and aging process. May be localized or generalized. May affect any joint and can occur at any age.

\section{Mechanism of pain in $\mathrm{OA}$}

Pain has been defined as "an unpleasant sensory and emotional experience associated with actual or potential tissue damage, or described in terms of such damage" (Merskey \& Bogduk 1994). Pain, as generally acknowledged, is mainly a signal that the body has been injured (Jenkins 1998).

Process of pain perception (Jenkins 1998, Bonica 1985, Raja et al 1999, Levine\& Reichling 1999)

- A noxious stimulus causes stimulation of nociceptors (pain receptors) in the receptor organ(e.g., joint)

- This firing of primary afferent fibres at the site of tissue injury causes axonal release of substance $\mathrm{P}$ (SP). This stimulation leads to activation of cells in the dorsal horn of the spinal cord and transmission of the nerve impulse to the midbrain and cortex. Thus, impulses traveling along first-order neuron synapse on second-order neuron in the dorsal horn of the spinal cord. The axon crosses to the contralateral side and ascend to synapse on the third-order neurons. The third-order neurons send fibres to the cerebral cortex where conscious perception of the sensation occurs.

- Transmission of sensory information is modulated (inhibit or potentiated) throughout the nervous system by neurons from the midbrain and spinal cord that release endogenous opioids, catecholamines and other neurotransmitters.

- Peripheral nociceptor sensitization which is the transmission of impulses at subnormal threshold occurs following the release of chemical mediators such as prostagladins and leukotrienes at the site of injury or damage.

- Continued stimulation by peripheral nociceptors then leads to sensitization of neurons in the spinal cord. This is known as central sensitization.

Tissue injury results in the release of inflammatory mediators such as serotonin, bradykinin, calcitonin gene-related peptide (CGRP) and substance $\mathrm{P}$ (SP) which lead to nociceptor nerve fibre sensitization in peripheral tissue. These damaged fibres release inflammatory agents causing a spread of increased sensitivity around the area of tissue damage. This is called primary hyperalgesia. The repeated depolarization of primary afferent fibres leads to a continual release of neurotransmitters onto the secondary neurons in the spinal cord, resulting in central sensitization and secondary hyperalgesia.

Recent evidence indicates that peripheral pain sensitization is a feature of osteoarthritis in joint (Farrel et al 2000, Bradley et al 2004). Farrel et al in 2000, conducted a study aimed at establishing the presence of hyperalgesia at the thumb in subjects with OA of the hand and also to explore the relationship between sensitivities to extrinsic stimuli and the experience of clinical pain. They hypothesized that in groups with painful OA of the first carpometacarpal (CMC) pain thresholds would be lower over the thumb relative to the forearm, a regional difference that would be absent in cases without pain. In addition they proposed that among cases with symptoms greater sensitivity at the thumb would be associated with more intense clinical pain. The major findings in the 80 subjects that underwent the psychophysical evaluation were a lower thermal and mechanical pain thresholds were evident over the thumb relative to the forearm in the groups with persistent pain, incident as well as movement pain. Patients with only incident pain, pain-free OA patients and pain free controls did not show any 
regional differences in sensitivity to thermal and mechanical pain thresholds. The findings in this study indicate that hyperalgesia over the thumb could be a consequence of spinal sensitization and $\mathrm{OA}$ in the hand is associated with local hyperalgesia which may be mediated through the dorsal root reflexes. The authors opined that this peripheral sensitization may be dependent on reflexes that are in turn dependent on spinal environment which is sensitized as well.

Kidd and colleagues in 2003, in their study aimed at comparing the contribution of SP to behavioral responses and inflammatory markers at serial time points in two chronic complete Freund`s adjuvant (CFA) models of inflammation using NK-1 receptor knockout mice demonstrated that mechanical hyperalgesia was significantly reduced in animals with a selective deletion of the NK-1 receptor for SP. They opined that SP which has been showed to have important influences on inflammatory and immune cell function may exert its peripheral pain sensitization through mediation by nerve growth factors or cytokines.

In another study, Lindh et al (1997) demonstrated increased SP-like activity in the cerebrospinal fluid (CSF) in patients with painful hips or knee OA compared to controls from the analysis of CSF samples from 11 patients. This finding has also been reported by Russell et al 1994. These findings suggest a gradual transition in OA from uncomplicated nociceptive pain to secondary sensory disturbances having similarities with observed findings in fibromyalgia.

In addition to peripheral pain sensitization, Melzack et al (2001) in their study aimed at evaluating central neuroplasticity and pathologic pain concluded that central pain sensitisation at the spinal or cortical level can occur in osteoarthritis. They suggest that pain in osteoarthritis, therefore, could be due to local and central sensitisation of pain, pathways resulting in normal stimuli becoming painful with inflammation being an important feature in the process of osteoarthritis.

Most of the substances involved in inflammation such as proinflammatory cytokines and bradykinins interact with the nociceptive fibres present within the joint and induce hyperalgesia and allodynia seen in patients with chronic inflammatory joint disease like

OA. These mechanisms acting in concert could participate in the progression of hyperalgesia to chronicity.

\section{Progression of OA to chronicity}

Bonica (1990) defines chronic pain (CP) as persisting a month beyond the usual course of an acute disease or a reasonable time period for an injury to heal.

$\mathrm{CP}$ differs from the acute process not only in the duration of its course but also the different receptors involved in the mechanisms of action for acute and CP. Those most involved in the acute process are a-amino-3-hydroxy-5-methylisoxazole-4-propionic acid (AMPA) receptors, while those of primary importance in the sensation of CP are N-methyl-D-aspartate (NMDA) receptors (Brookoff 2000). Activation of NMDA receptors causes the release of peptide neurotransmitter SP which amplifies the pain by causing the spinal neurons carrying the pain to be easily stimulated. Elevated levels of SP in spinal fluids have been documented in patients with OA and fibromyalgia (Russell et al 1994, Lindh et al 1997).

The progression of nociception from an acute to a chronic process has yet to be fully understood. However, recent evidence from animal experiments as well as human research suggest that peripheral mechanisms in acute pain and longterm potentiation (LTP) of neuronal sensitivity to nociceptive inputs in the dorsal horn of the spinal cord may underline the transition from acute to a chronic process.

The important role of peripheral mechanisms in CP associated with chronic inflammation is suggested by the efficacy of aspirin and nonsteriodal anti-inflammatory agents, whose action is predominantly peripheral.

LTP in spinal nociceptive systems has been suggested as one of the mechanisms underpinning the transition of acute into $\mathrm{CP}$.

The pioneering work of Bliss and Lømo (1973) and Lømo (2003) showed that brief highfrequency trains of electrical stimuli resulted in increased efficiency of transmission at the hippocampus of the rabbit that could last for 
hours. This phenomenon was described as LTP. Recently, this observation has been extended to humans by Klein et al (2004) who suggested that the generation of LTP may be one mechanism whereby acute pain may be transformed into a CP state. It seems possible that LTP may underlie some forms of afferent induced hyperalgesia and simultaneous activation of NMDA, SP neurokininI (NK-I) and glutamate receptors are required for the induction of spinal LTP. Thus, it is likely that the conditioning stimuli that induce synaptic LTP in the superficial spinal dorsal horn are similar to those that trigger hyperalgesia. LTP is likely to occur in both the sensory and the affective pain pathways. Additionally, spinal LTP and injury induced hyperalgesia share signal transduction pathways, which make use dependent LTP an attractive model of injury induced central sensitization and hyperalgesia (Sandkühler 2000, Sandkühler et al 2000, Liu and Sandkühler 1998). Extensive studies have shown that wide dynamic range (WDR) neurons are present in the deeper laminae of the spinal cord (lamina IV-VI) where nociceptive specific neurons are prevalent (Bester et al 2000). Studies have also shown that the deep WDR cells have the ability to code noxious and innocuous stimuli and these neurons may play a pivotal role in transmission of painful inputs (Suzuki et al 2002). Furthermore, Afrah et al in 2002 while using microdialysis to analyse the cerebrospinal fluid in the spinal cord, demonstrated that the release of SP is increased during the high-frequency stimuli (HFS). The result of the study indicate that SP and NK-I receptors play a significant role in the induction of LTP in the deep WDR neurons.

Svendsen et al (1998) and Svendsen et al (1999) demonstrated in these studies that AMPA and NMDA receptor antagonists blocked the induction of LTP and NMDA receptor blockade depotentiated the established LTP respectively. Thus it is apt to conclude that the activation of glutamate, AMPA, NMDA and the NK-I receptors is crucial to the induction of LTP in the deep WDR neurons.

Taking these data into a wider context and given the fact that LTP is considered not only as a cellular and synaptic model for memory and learning but also the fact that LTP can be induced throughout the central nervous system, it will not be simplistic to conclude that LTP in the spinal nociceptive systems constitutes one of the most likely single cellular mechanisms to explain how acute pain may progress to CP (Jacobson et al 2001).

While the search for a broader and comprehensive understanding of the mechanism of transition from acute to CP continues, Brookoff (2000) submit that repetitive generation of pain signals alter the neural pathways making them hypersensitive. This pain signal becomes incorporated into the spinal cord, replaying like a needle stuck on a record album. This simplifies the proposed mechanisms of a simple nociceptive inputs becoming persistent pain.

\section{Implication for practice}

The vast body of data and research findings over the last decade has led to a better understanding of the neurophysiology of pain. This invariably is beginning to influence clinical practice. For instance anti-inflammatory agents that prevent or reduce peripheral sensitization cannot be neglected as an important adjunct in comprehensive pain management. The important role of peripheral mechanisms in $\mathrm{CP}$ associated with chronic inflammation is suggested by the efficacy of aspirin and nonsteriodal anti-inflammatory agents, whose action is predominantly peripheral.

Again pain medication are now prescribed and given as scheduled regimens instead of the hitherto clinical practice of ordering pain medication on an as-needed basis. Furthermore, severity of chronic pain and its associated complications have been found to be greatly reduced when more potent analgesics are started earlier.

Thus, substantial pain control for some patients has been achieved by using sustainedrelease opiate preparations aimed at reducing breakthrough pain and associated adverse effects (Hale et al 1999). Because of the present understanding of the neurophysiology of pain and because of the findings that opiates have both peripheral and central analgesic effects, opiates are now been used in conditions such as arthritis in 
which they had previously been avoided.

The use of gabapentin, an effective anticonvulsant for the treatment of neuropathic pain is due to the fact that gabapentin can enhance GABA activity and possibly prevent glutamate release (Rosenberg et al 1997). Further research may provide a spectrum of agents with glutamate receptor blocking activity in the nearest future.

As discussed above, windup phenomenon has become the focus of recent research in pain management with the hope of finding a drug that will modulate this phenomenon in order to prevent its long-term sequelae. CP has been known to be modulated by noradrenaline and serotonin reuptake blocking agents. The awareness that increased concentrations of these neurotransmitters in the central nervous system inhibit pain impulse transmission has led to a search for other agents with similar actions.

The tremendous strides that have been achieved in the search for better care of patients with pain should serve as a springboard for the jump from animal research model to intergrated care model in humans. It is hoped that intergrated care and effective therapy will mitigate the longterm sequalae of persistent nociception on the central nervous system.

\section{Conclusion}

It is clear from the foregoing that any simple unitary concept about the link between joint damage and symptoms in osteoarthritis is untenable. We are faced with a complex interaction between local events in the joint, pain sensitization, the cortical experience of pain, and what people are doing in their everyday lives.

There is a vast body of evidence to suggest that $\mathrm{OA}$ is a heterogenous condition that involved not only the articular cartilage but also an adaptive response of the bone and the synovium to a variety of environmental, genetic and biomechanical stresses (Denko et al 1996, Pelletier et al 1996, Westacott et al 1996, Fink et al 2001, Volck et al 2001, Yuan et al 2004, Shibakawa et al 2005).

There is also growing evidence pointing towards LTP as the most likely mechanism for the transition of acute nociception to a CP state.

The complexity and plasticity of the nociceptive system not only serves survival needs but also provides research opportunities for pharmacologic modulation of human suffering. Pain relief must always been viewed as humane. $\mathrm{CP}$ puzzles patients because they are unable to comprehend why modern medicine cannot find a solution to their problem. To the health care provider CP is a frustration because the etiology is almost unclear and satisfactory treatment is elusive [51-55]. Carron's assertion that "minimal pathology with maximum dysfunction remains the enigma of CP” epitomizes the challenge facing health care provider.

\section{REFERENCES}

Abramson SB: (2004): Inflammation in osteoarthritis. J Rheumatol Suppl 2004; 70:70-76. Afrah AW, Fiska A, Gjerstad $J$ et al (2002): Spinal substance $P$ release in vivo during the induction of long-term potentiation in dorsal horn neurons. Pain 2002; 96:49-55.

Agel et al. The burden of musculoskeletal conditions at the start of the new millennium. Geneva. World Health Organisation 2000.

Alvarez FJ, Fyffe RE (2000): Nociceptors for the $21^{\text {st }}$ century. Curr Rev Pain 2000; 4: 451-458.

Berne RM, Levy MN (1998): ed. Physiology. $4^{\text {th }}$ ed. St. Louis, MO; Mosby, 1998

Bester H, Chapman V, Besson JM et al (2000): Physiological properties of the lamina I spinoparabrachial neurons in the rat. $J$ Neurophysiol 2000; 83: 2239-2259.

Bliss TV, Lømo T (1973): Long-lasting potentiation of synaptic transmission in the dentate area of the anaesthetized rabbit following stimulation of the perforant path. J Physiol 1973; 232:331-356.

Bonica JJ (1985): History of pain concepts and pain therapy. Seminars in Anaesthesia 1985; 4:189-219.

Bradley LA, Kersh BC, DeBerry JJ et al (2004):. Lessons from fibromyalgia: abnormal pain sensitivity in knee osteoarthritis. Novartis found symp 2004; 260: 258-70; discussion 270-79. Brookoff D. Chronic pain: 1. A new disease? Hosp Pract2000; 35:45-59. 
Brooks P (2003):. Inflammation as an important feature of osteoarthritis. Bull World Health Organ 2003; 81:689-90.

Creamer P, Hunts M, Dieppe P (1996): Pain mechanisms in osteoarthritis of the knee: effect of intraarticular anesthetic. $J$ Rheumatol 1996; 23:1031-36.

Denko CW et al. Growth factors, insulin-like growth factors- 1 and growth hormone in synovial fluid and serum of patients with rheumatic disorders. Osteoarthritis and Cartilage 1996; 4: 245-249.

Dye SF, Chew MH (1993): The use of scintigraphy to detect increased osseous metabolic activitiy about the knee. J Bone Joint Surg Am 1993; 75:1388-1406.

Farrel M, Gibson S, McMeeken J et al. (2000) Pain and hyperalgesia in osteoarthritis of the hands. J Rheumatol 2000; 27:441-47.

Felson DT, Chaisson CE, Hill CL et al. (2001): The association of bone marrow lesions with pain in knee osteoarthritis. Ann Intern Med 2001; 134:541-549.

Felson DT, Mclaughlin S, Goggins $J$ et al. (2003) Bone marrow edema and its relation to progression of knee osteoarthritis. Ann Intern Med 2003; 139:330-36

Fink $\mathrm{C}$ et al. The effect of dynamic mechanical compression on nitric oxide production in the meniscus. Osteoarthritis and Cartilage 2001; 9: 481-487.

Ganong WF (2001):. Review of medical physiology. $20^{\text {th }}$ ed. McGraw-Hill Companies Inc. 2001.

Hale ME, Fleischmann R, Salzman R, et al (1999):. Efficacy and safety of controlled-release versus immediate-release oxycodone: Randomized, double-blind evaluation in patients with chronic back pain. Clin J Pain 1999; 15:179183.

Jacobson L, Mariano AJ (2001):. General considerations of chronic pain. In: Loeser JD, Butler SH, Chapman CR, Turk DC, eds. Bonica's Management of Pain. Philadelphia, PA: Lippincott Williams \& Wilkins; 2001:241-254.

Jenkins BJ (1998):. Physiology of pain: Module 2 Lecture. In: PGCert/PGDip/MSc in Pain Management. Cardiff University, Wales,. 1-53.
Kellgren JH, Samuel EP (1950):. The sensitivity and innervation of the articular capsule. $J$ Bone Joint Surg Br; 32: 84-92.

Kidd BL et al. Inflammation and hyperalgesia in NK-1 receptor knock-out mice. Neuroreport 2003; 14: 2189-9.

Klein T, Magerl W, Hopf HC et al (2004): Perceptual correlates of nociceptive long-term potentiation and long-term depression in humans. J Neurosci; 24:964-971.

Lawson SN. (2002): Phenotypes and function of somatic afferent nociceptive neurons with C, Adelta- or Aalpha/beta fibres. Exp Physiol; 87:239-244.

Lindh C, Liu Z, Lyrenas S et al (1997): Elevated cerebrospinal substance P-like immunoreactivity in patients with painful osteoarthritis, but not in patients with rhizopatic pain from a herniated lumbar disc. Scan J Rheumatol; 26:468-472.

Liu XG, Sandkühler J. (1998): Activation of spinal NMDA or neurokinin receptors induces long-term potentiation of spinal C-fibre-evoked potentials. Neurosci 1998; 86:1209-1216.

Lømo T (2003): The discovery of long-term potentiation. Philos Trans $R$ Soc Lond B Biol Sci 2003; 358: 617-620.

Martini FH (1998): Articulations: In: Fundamentals of anatomy and physiology. $4^{\text {th }}$ ed. Prentice Hall; Inc. New Jersey 1998.

Melzack $\mathrm{R}$ et al. Central neuroplasticity and pathological pain. Ann NY Acad sci 2001; 933: 157-74.

Merskey H, Bogduk N (1994): Classification of chronic pain: Description of chronic pain syndromes and definitions of pain terms. Seattle, WA: International Association for the Study of Pain Press

Murray CJL (1996): The global burden of disease. Geneva: World Health Organisation1996.

Pelletier J-P et al. The increased synthesis of inducible nitric oxide inhibits IL-Ira synthesis by human articular chondrocytes: possible role in osteoarthritic cartilage degradation. Osteoarthritis and Cartilage 1996; 4: 77-84.

Rawlinson SC, Pitsillides AA, Lanyon LE (1996): Involvement of different ion channels in osteoblasts` and osteocytes' early responses to mechanical strain. Bone 1996; 19: 609-14. 
Reginster JY. The prevalence and burden of arthritis. Rheumatology 2002; 41 (suppl.): 3-6.

Rehman Q, Lane NE. Getting control of osteoarthritis pain: an update on treatment options. Postgrad Med 1999; 106: 127-134.

Romanes GJ. General introductions; Joints. In: Cunningham's Manual of practical anatomy. $15^{\text {th }}$ ed. Vol (1). Oxford: Oxford University Press1986. Rosenberg JM, Harrell C, Ristic H et al. The effect of gabapentin on neuropathic pain. Clin J Pain 1997; 13:251-255.

Russell IJ, Orr MD, Littman B et al. Elevated cerebrospinal fluid levels in patients with fibromyalgia syndrome. Arthritis Rheum 1994; 37: 1593-1601.

Sandkühler J, Benrath J, Brechtel C et al.Synaptic mechanisms of hyperalgesia. Prog Brain Res 2000; 129: 81-100.

Sandkühler J. Learning and memory in pain pathways. Pain 2000; 88: 113-118.

Sherrington CS (1947):. The integrative action of the nervous system ed 2. New Haven, Yale University Press, 1947. Pp1-147.

Shibakawa A et al.The role of subchondral bone resorption pits in osteoarthritis: MMP production by cells derived from bone marrow. Osteoarthritis and Cartilage 2005; 13: 679-687.

Snell RS (2004): Basic Anatomy. In: Clinical Anatomy. $7^{\text {th }}$ ed. Lippincott Williams \& Wilkins

Sowers MF, Hayes C, Jamadar D et al (2003). Magnetic resonance-detected subchondral marrow and cartilage defect characteristics associated with pain and x-ray defined knee osteoarthritis. Ostoearthritis cartilage 2003; 11: 387-393.

Suzuki R, Morcuende S, Webber $M$ et al (2002): Superficial NK-I expressing neuron control spinal excitability through activation of descending pathways. Nat Neurosci 2002; 5: 1319-1326.
Svendsen F, Tjølsen A, Hole K (1998): AMPA and NMDA receptor-dependent spinal LTP after nociceptive titanic stimulation. Neuroreport 1998; 9: 1185-1190.

Svendsen F, Tjølsen A, Rygh LJ et al. (1999): Expression of lon-term potentiation in single wide dynamic range neurons in the rat is sensitive to blockade of glutamate receptors. Neurosci Lett 1999; 259: 25-28.

Townes AS (1999): Osteoarthritis. In: Barker LR, Burton JR, Zieve PD, editors. Principles of ambulatory medicine. $5^{\text {th }}$ ed. Baltimore: Williams \& Wilkins; 1999. p. 960-73.

van den Berg W, van der Kraan PM, van Beuningen HM (2003): Synovial mediators of cartilage damage and repair in osteoarthritis. In: Brandt KD, Doherty M, Lohmander LS, eds. Osteoarthritis. $2^{\text {nd }}$ edn. Oxford: Oxford University Press, 2003:147-55.

Volck B et al. Studies on YKL-40 in knee joints of patients with rheumatoid arthritis and osteoarthritis. Involvement of YKL-40 in the joint pathology. Osteoarthritis and Cartilage 2001; 9: 203-214.

Westacott CI, Sharif M (1996): Cytokines in osteoarthritis: mediators or markers of joint destruction? Semin Arthritis Rheum 1996; 25:254272.

Wojtys EM, Beamann DN, Glover RA et al (1990). Innervation of the human knee joint by substance-P fibers. Arthroscopy 1990; 6: 254-263. Wyke B. The neurology of joints: a review of general principles. Clin Rheum Dis 1981; 57:233239.

Yuan G-H et al.Characteristics of cells from pannus-like tissue over articular cartilage of advanced osteoarthritis. Osteoarthritis and Cartilage 2004; 12: 38-45. 